\title{
Comparison of high-frequency transabdominal ultrasonography and spiral computed tomography with colonoscopy in diagnosis of colon cancer
}

\author{
Sumnyan W. ${ }^{1}$, Komut O. $^{2}$, Ligu L. ${ }^{3}$ \\ ${ }^{1}$ Dr. Wangju Sumnyan, Assistant Professor, Department of Radiology \& Imaging, Tomo Riba Institute of Health and \\ Medical Sciences (TRIHMS), Naharlagun, Arunachal Pradesh, ${ }^{2}$ Dr. Ojing Komut, Assistant Professor, Department of \\ Surgery, Tomo Riba Institute of Health and Medical Sciences (TRIHMS), Naharlagun, Arunachal Pradesh, ${ }^{3}$ Dr. Leena \\ Ligu, Consultant Gynaecologist, RK Mission Hospital, Itanagar, Arunachal Pradesh.
}

Corresponding Author: Dr. W. Sumnyan, Assistant Professor, Department of Radiology \& Imaging, Tomo Riba Institute of Health and Medical Sciences (TRIHMS), Naharlagun, Arunachal Pradesh. Email: sumnyanwang@gmail.com,dr.aridita@gmail.com

\begin{abstract}
Objective: To compare high-frequency transabdominal ultrasonography (USG) and spiral computed tomography (CT) with colonoscopy in diagnosis of colon cancer. Design: A prospective comparative study of accuracy of USG and CT scan with colonoscopy. Subjects: Sixty patients with a clinical suspicion of colon cancer after a detailed clinical history and a thorough clinical examination were included. Patients with a known diagnosis of colon cancer or in whom histopathological diagnosis could not be established were excluded. Methods: All 60 patients who met the inclusion and exclusion criteria underwent transabdominal USG, CT scan-abdomen \& pelvis, followed by colonoscopy. The CT and USG scans were reported by different radiologists without previous knowledge of any findingsof the other test or of the subsequent colonoscopy. The colonoscopy was performed by different clinicians, none of whom was aware of the USG or CT diagnosis. Result: Colonoscopy diagnosed 29 patients with colon cancer out of 60 enrolled patients. USG detected colon cancer in all the 29 patients with a sensitivity of $100 \%$ and a specificity of $87.1 \%$. CT scan diagnosed colon cancer in all the 29 patients with a sensitivity of $100 \%$ and a specificity of $74.2 \%$. Conclusions: Colonoscopy is still necessary when a suspicious lesion is identified. However, CT and USG can screen out suspected patients who can be subsequently referred for colonoscopy. This would reduce the need for colonoscopy in a large proportion of clinically suspected patients andalso avoidan invasive procedure like colonoscopy as first line investigation in elderly patients suspected of having colonic cancer.
\end{abstract}

Key words: Colorectal cancer, Colonoscopy, CT scan, Ultrasonography.

\section{Introduction}

Colorectal cancer (CRC) accounts for $10 \%$ of all tumour types world wide. It is the third most common tumour in men and the second in women with higher in males (ratio: 1.4). There is a 10-fold difference in incidence between several regions across the globe. $\mathrm{CRC}$ is the fourth most common cancer-related cause of death ( $\sim 8 \%$ of all cancer deaths), in the world [1,2]. A rapid increase in both CRC incidence and mortality are now observed in many medium-to-high HDI countries particularly in Eastern Europe, Asia and South America [2].

Manuscript received: $20^{\text {th }}$ August 2018

Reviewed: $30^{\text {th }}$ August 2018

Author Corrected: $6^{\text {th }}$ September 2018

Accepted for Publication: $11^{\text {th }}$ September 2018
Malignant lesions arise from a preexisting adenoma in many cases. The American Cancer Society has published recommendations for CRC screening according to the stratification of individuals based on risk. Accordingly, patients are categorized into three groups: average (age $\geq 50$ years), moderate (family history of CRC, personal history of small adenomatous polyps or CRC) or high risk (inflammatory bowel disease, family history of familial adenomatous polyposis or nonpolyposis colon cancer).

In spite of the availability of numerous tests for CRC screening for detecting early stage cancer or adenomas, there are limitations attached to each procedure. 


\section{Original Research Article}

Colonoscopy is one of the screening tests and is very popular as it has the advantages of direct visualization of the colonic mucosa and having the option of taking a biopsy directly from the polyps or lesions. However, colonoscopy is invasive and require a rigorous bowel preparation which is frequently unsatisfactory and distressing, especially in the geriatric population. They often require admission prior to the investigation, which adds up to the expense. Furthermore, the results are often inconclusive in the geriatric population due to retained faeces or incontinence.

It fails to visualize the caecum in $5-10 \%$ of cases. Computed tomography (CT) colonography is minimally invasive but has the limitation of a cumulative radiation dose when repeatedly performed. In spite of certain advantages of requiring no sedation and being noninvasive, current clinical practice guidelines do not include specific recommendations on CT colonography for screening. It needs to be established whether CT colonography is preferable to colonoscopy in this context [3,4].

A detailed transabdominal USG examination forms an essential part of the investigation of the abdomen. USG screening can help in the diagnosis of large bowel diseases like colonic cancer or diverticulosis. It is a safe, widely available, cheap, noninvasive imaging technique.

It allows real-time examination of the abdomen without the use of any radiation and can be performed at any time. It has a disadvantage that the evaluation of the bowel depends more on the operator experience and expertise than the sonographic evaluation of other abdominal organs [5].

This study was conducted to determine the accuracy of high-frequency transabdominal USG and CT colonography compared with colonoscopy in the diagnosis of colon cancer.

\section{Aims and Objectives}

To determine the sensitivity and specificity of highfrequency transabdominal ultrasonography and Computed tomography in the diagnosis of colon cancer compared with Colonoscopy.

\section{Materials \& Methods}

A prospective comparative study was done at the Department of Radiology, RK Mission Hospital, Itanagar, Arunachal Pradesh to compare the sensitivity and specificity of high-frequency transabdominal ultrasonography and CT scan with colonoscopy in the diagnosis of colon cancer in patients clinically suspected of having the disease from September 2016 to June 2018.

A total of 60 patients were included in the study sample. Inclusion and exclusion criteria were as follows:

Inclusion criteria- Patients with a clinical suspicion of colon cancer after a detailed clinical history and a thorough clinical examination. The major clinical complaints included history of alteration in bowel habits, melena, rectal bleeding, abdominal cramps, presence of abdominal lump, and other complaints specific for colon cancer. The clinical examination stressed on the presence of palpable abdominal lump.

Exclusion criteria- Patients with a known diagnosis of colon cancer or in whom histopathological diagnosis could not be established were excluded from the study.

All 60 patients who met the inclusion and exclusion criteria underwent transabdominal sonography, CT scan-abdomen \& pelvis, followed by colonoscopy. The CT and USG studies were reported by different radiologists without knowledge of the findings of the other study or of the subsequent colonoscopy. The colonoscopy was performed by a number of different clinicians, none of whom was aware of the USG or CT diagnosis.

Institutional ethics committee approval was obtained before the study. An informed, bilingual, written consent was obtained before including the patients as study subjects.

Procedure- A standard ultrasound scanner (ACUSON X300, SIEMENS) with a selection of transducers from 3.0 to $10.0 \mathrm{MHz}$ was used for the transabdominal ultrasonography in all patients. A complete abdominal and pelvic scan was performed using appropriate low frequency transducers followed by specific examination of the colon using the high-frequency transducers. An attempt was made in all the patients to identify the whole extent of the colon with slight compression wherever necessary. The sonographic criteria used for diagnosis of a possible colon carcinoma were [1] a localized and irregular thickening of the colonic wall with heterogenous low echogenicity [2], an irregular contour [3], a lack of demonstrable movement or change of configuration of the bowel on real-time scanning, and [4] absence of wall stratification [6]. 
CT scan was performed using DEFINITION PERSPECTIVE 128 SLICE (SIEMENS, GERMANY) with $5 \mathrm{~mm}$ thick slices at a pitch of 1.5 through the whole abdomen and pelvis. A total of $60 \mathrm{ml}$ of iodinebased IV contrast agent $(60 \%)$ was administered manually just before the start of the CT scan. Neither rectal air nor contrast medium was used, as this would have been unpleasant for the patient, and introduced the

\section{Original Research Article}

problem of incontinence. The diagnostic appearance of colonic cancer was, typically, of a circumferential or focal mass with an uneven, lobulated configuration [7].

The patient then underwent routine bowel preparation with colonoscopy performed the following day. A colonoscopy diagnosis of malignancy was confirmed by biopsy.

\section{Results}

This prospective study was done between September 2016 to June 2018 and enrolled 60 patients during this period. All these 60 patients with clinical suspicion of colon cancer underwent ultrasonography, computed tomography and colonoscopy.

Of the 29 patients who had colon cancer, $18(62 \%)$ were males and $11(38 \%)$ were females. The age range of patients varied from 19 years to 80 years. The mean age of the patients was 52.2 years [Table 1 ].

Table-1: Age distribution among subjects.

\begin{tabular}{|c|c|}
\hline Age group & No. of cases \\
\hline$<20$ yrs & 1 \\
\hline $20-30$ yrs. & 2 \\
\hline $30-40$ yrs & 5 \\
\hline $40-50$ yrs & 4 \\
\hline $50-60$ yrs. & 7 \\
\hline $60-70$ yrs & 4 \\
\hline$\geq 71$ yrs & 6 \\
\hline
\end{tabular}

Table-2: Site distribution of colon cancer among subjects

\begin{tabular}{|c|c|c|}
\hline Site & No. of cases & Percentage \\
\hline Cecum & 3 & $10.3 \%$ \\
\hline Ascending colon & 5 & $17.2 \%$ \\
\hline Hepatic flexure & 1 & $3.4 \%$ \\
\hline Transverse colon & 4 & $14 \%$ \\
\hline Splenic flexure & 1 & $3.4 \%$ \\
\hline Descending colon & 2 & $7 \%$ \\
\hline Sigmoid colon & 4 & $14 \%$ \\
\hline Recto sigmoid region & 9 & $31 \%$ \\
\hline Total & $\mathbf{2 9}$ & $\mathbf{1 0 0} \%$ \\
\hline
\end{tabular}

Table-3: Comparison of $\mathrm{Ct}$ and USG detection of colon cancer.

\begin{tabular}{|c|c|c|c|c|c|c|c|c|}
\hline Investigation & $\begin{array}{c}\text { True } \\
\text { +ve }\end{array}$ & $\begin{array}{c}\text { True } \\
\text {-ve }\end{array}$ & $\begin{array}{c}\text { False } \\
\text { +ve }\end{array}$ & $\begin{array}{c}\text { False } \\
\text {-ve }\end{array}$ & $\begin{array}{c}\text { Sensitivity } \\
\%\end{array}$ & $\begin{array}{c}\text { Specificity } \\
\mathbf{\%}\end{array}$ & $\begin{array}{c}\text { PPV } \\
\%\end{array}$ & $\begin{array}{c}\text { NPV } \\
\%\end{array}$ \\
\hline CT & 29 & 23 & 8 & 0 & $100 \%$ & $74.2 \%$ & $78.4 \%$ & $100 \%$ \\
\hline USG & 29 & 27 & 4 & 0 & $100 \%$ & $87.1 \%$ & $87.9 \%$ & $100 \%$ \\
\hline
\end{tabular}

The most common clinical presentation was that of an alteration in bowel habits with predominance of constipation (90\%). The other major complaints included melena, abdominal cramps, and rectal bleeding. 
Colonoscopy was significantly abnormal in 30 of the 60 patients $(50 \%)$. Colon cancer was visually diagnosed by colonoscopy in 29 of these patients and malignancy was confirmed by histology in all cases (48.3\%). One patient had features suggestive of ulcero-proliferative colitis, which on histology was shown to have nonspecific colitis. Colonoscopy could be passed beyond the visualized growth in only 9 of the 29 patients who had colon cancer. Large obstructive growth prevented the passage of the colonoscope proximally in the 20 patients.

The commonest presentation of colon cancer on ultrasonography was that of an echo poor focal/asymmetrical bowel thickening. Ultrasonography correctly diagnosed colon cancer in all the 29 patients with no false negatives but four false positives. In one false positive case deserves ultrasonography erroneously suggested a growth involving the sigmoid colon. This turned out to be a growth arising from the small bowel and the histologic diagnosis of the operated specimen proved to be suggestive of leiomyosarcoma.

Computed tomography correctly diagnosed colon cancer in all the 29 cases. There were no false negatives but eight false positives. Three false positives were in the cecum, two in the ascending colon and three in the rectosigmoid region, suggesting that particular care is needed in interpreting the bowel in these regions. The commonest CT presentation of colon cancer was that of an asymmetrical wall thickening occurring in $25(86 \%)$ patients. Focal mass was the presentation in the other $5(14 \%)$ patients. CT also correctly detected the small bowel mass that turned out to be leiomyosarcoma.

On ultrasonography, the wall thickness of the colon growth varied from $0.9 \mathrm{~cm}$ to $6.5 \mathrm{~cm}$, with a mean wall thickening of $2.0 \mathrm{~cm}$. The wall thickness on Computed tomography ranged from $1.1 \mathrm{~cm}$ to $6.5 \mathrm{~cm}$, with a mean value of $2.2 \mathrm{~cm}$. The mean value of wall thickening in colon cancer appeared more on CT than on ultrasonography by $0.2 \mathrm{~cm}$.

There were thus 29 colonoscopically diagnosed and histologically proven cancers in the 60 patients who had all three investigations completed. Nine were in the recto sigmoid region, four at the sigmoid colon, two in the descending colon, one in the splenic flexure, four in the transverse colon, one in the hepatic flexure, five in the ascending colon, and three in the cecum [Table 2].

Ultrasonography detected colon cancer in all the 29 patients with a sensitivity of $100 \%$ and a specificity of $87.1 \%$. USG provided additional useful information in five patients. Computed tomography diagnosed colon cancer in all the 29 patients with a sensitivity of $100 \%$ and a specificity of $74.2 \%$. CT provided additional useful information in six patients [Table 3].

\section{Discussion}

Several studies discussing the use of transabdominal USG in the diagnosis of colonic cancer have been published. Some advocated the limited use of USG in specific situations, for example as a supplement to barium enema where the cecum was poorly seen [8] or in the Accident and Emergency department $[9,10]$.

Jeremy Price and Constantine Metreweli, in 1988 concluded that ultrasound is a useful primary diagnostic technique for colonic neoplasms, with a predictive value of $79 \%$ in detecting clinically non-palpable lesions. This study involved 1700 abdominal ultrasound scans over a period of 2 years, including a search for bowel disease in the scanning routine [11].

In another study, Shirahama et al [6] in 1994 described four sonographic patterns that allowed correct diagnosis of colonic carcinoma in $90 \%$ of patients as used in this study and described in the methods in this paper.
Colonoscopy was used to confirm the USG diagnosis of colonic cancer in the study. Several studies have used orally or rectally introduced water in an attempt to improve visualization of the colon. The single study using oral administration of water, termed "sonoenterocolonography", was performed on 56 patients who had already undergone BE, followed by colonoscopy in those with abnormal findings [12].

This gave a sensitivity for lesions larger than $10 \mathrm{~mm}$ of $89 \%$ but has not been repeated. Two studies used rectally introduced water, termed "hydrocolonicsonography" [13, 14]. Despite giving good results, this involves colonic instillation of up to $1500 \mathrm{ml}$ of water, which strongly decreases the advantage of this technique in the elderly population. The larger of these studies [14] compared transabdominal USG, hydrocolonic-sonography and colonoscopy. The specificity of transabdominal USG for the detection of 


\section{Original Research Article}

colon cancer was $99 \%$ and sensitivity was $33 \%$. The results of hydrocolonic-sonography appear very good, with a sensitivity for cancer of $97 \%$ and of $91 \%$ for polyps of $7 \mathrm{~mm}$ or greater in diameter. Unfortunately, these results were not duplicated in another study [15] which compared hydrocolonic-sonography with colonoscopy and found a sensitivity for cancer detection of $0 \%$ and for polyps of $7 \mathrm{~mm}$ or greater of $12.5 \%$. The marked discrepancy between the two centres is difficult to explain but, even if the accuracy of the technique were confirmed, it is just as invasive as barium enema (BE) with its attendant problems in the elderly.

W K Loftus et al. in 1999 concluded that both transabdominal ultrasound and $\mathrm{CT}$ are possible alternatives to colonoscopy in the investigation of symptomatic patients with suspected colonic cancer. USG had a sensitivity and specificity of $100 \%$ and CT a sensitivity of $100 \%$ and a specificity of $84 \%$. USG and CT were poor at detecting polyps. If the detection of polyps greater than $2 \mathrm{~cm}$ is included, then USG sensitivity falls to $67 \%$ and CT sensitivity falls to $89 \%$ and specificity rises to $88 \%$ [16].

Transabdominal USG in our study detected colon cancer in all the 29 patients with a sensitivity of $100 \%$ and a specificity of $88.6 \%$. It also demonstrated the adjacent visceral invasion and provided other useful information in 5 patients.

A number of studies have used CT in the detection of colonic cancer. A retrospective review of pre-operative CT using an early generation scanner showed a sensitivity of $84 \%$ in detecting histologically proven cancers [7]. Two studies from one centre specifically applied the use of CT to the elderly. BE was used as the gold standard in one study [14], which found that CT had a sensitivity of $100 \%$ and specificity of $86 \%$ in detecting cancer, very similar to our study. From the study, J. J. Day et al. in 1993, concluded that CT should be the initial investigation of the large bowel in the frail elderly patients requiring inpatient bowel preparation; the more unpleasant $\mathrm{BE}$ could be reserved for those cases where $\mathrm{CT}$ is equivocal or severe symptoms are unexplained [4]. CT alone was performed in a second study from the same centre [17].

None of the patients with normal CT had confirmatory BE or colonoscopy and nor did over half of those thought to have cancer. As a result, it is not possible to make any statistical assessment from this study. The technique used in these two studies, however, had considerable advantages for the elderly patient in that only oral contrast medium was used without routine IV contrast medium or rectal contrast medium or air. Bowel preparation was not used in either of the studies.

W K Loftus et al. in 1999 concluded that both transabdominal ultrasound and $\mathrm{CT}$ are possible alternatives to colonoscopy in the investigation of symptomatic patients with suspected colonic cancer. USG had a sensitivity and specificity of $100 \%$ and CT a sensitivity of $100 \%$ and a specificity of $84 \%$. USG and $\mathrm{CT}$ were poor at detecting polyps. If the detection of polyps greater than $2 \mathrm{~cm}$ is included, then USG sensitivity falls to $67 \%$ and CT sensitivity falls to $89 \%$ and specificity rises to $88 \%$ [18].

Okizuka $\mathrm{H}$, et al. in 1995 demonstrated cancer in $89 \%$ of cases, using ultrafast CT with IV contrast medium [19].

Two studies in 1996 introduced the use of CT colonography as a technique in the evaluation of the colon [20,21]. The results in both studies were promising but the use of a bowel preparation and problems in retaining the air means that they are poorly suited to the elderly population. Computed tomographic (CT) colonography is a rapidly evolving technique that enables two- and three-dimensional views of the surface of the colon. In current clinical practice, CT colonography is used to evaluate those segments of the colon that are not visible as a result of incomplete colonoscopy and also to evaluate the colon proximal to an obstructing carcinoma [22, 23, 24].

Our study used both iodinated oral contrast (2\% to $3 \%$ ) and iodine-based intravenous contrast medium (60\%). Full opacification of the colon was achieved in only $60 \%$ of the patients. The oral contrast medium could be taken over a longer period with improved bowel opacification, ideally by starting the oral contrast preparation the night before the CT scan was performed. Bowel opacification can also be improved by administrating gastric hurrying agents like metoclopramide along with the oral contrast medium.

The problem of incontinence did not occur as neither rectal contrast medium nor air was used. No serious adverse reaction was reported with the use of IV contrast medium. However, $3(5 \%)$ of the 60 patients complained of vomiting and $2(3.3 \%)$ had amild rash. CT detected all cancers found at colonoscopy with a sensitivity of $100 \%$ and a specificity of $77.5 \%$. False positives were primarily due to faecal material giving the impression of a mass lesion. 


\section{Original Research Article}

Colonoscopy, itself, is not adequate in all the patients. In patients having consecutive colonoscopies on the same day, the second study found polyps larger than 1 $\mathrm{cm}$, which was missed on the first colonoscopy, in $6 \%$ [25].

\section{Conclusion}

This study shows that transabdominal USG and CT are useful in the diagnosis of colonic cancer in symptomatic patients. It supports the previous studies that demonstrated the suitability of these radiological investigations in the evaluation of colonic cancer.

Both techniques avoid the bowel preparation and the possible admission required for colonoscopy or $\mathrm{BE}$ in the elderly. CT has the disadvantage of a higher false positive rate. Both the techniques were sensitive for the mean diameter of cancers diagnosed in this study but smaller lesions may well be missed. It is not suggested that either technique is useful for screening asymptomatic patients but, although numbers were relatively small, this study showed good results in diagnosing colonic cancers in those patients with symptoms. Review of the combined findings of USG and CT with the occasional repeat study, should further improve results. Colonoscopy is still necessary when a suspicious lesion is identified.

However, CT and USG can screen out suspected patients who can then be referred for colonoscopy. This would reduce the need for barium enema and colonoscopy in a large proportion of clinically suspected patients and yet no carcinoma would be missed. This implies that significant savings could be made by using USG or CT rather than colonoscopy as the first line investigation in elderly patients suspected of having colonic cancer.

Relevance of the Study: This study re-emphasizes the roles of CT scan and ultrasound in diagnosis of colon cancer in symptomatic patients in a resource limited settings. Very few studies have been conducted in remote centers with limited resources. This study justifies the usage of CT scan and USG in these settings.

\section{Authors contribution}

1. Dr Wangju Sumnyan: Study concept and design and investigator of the study.

2. Dr OjingKomut: Study concept and design, coinvestigator

3. Dr Leena Ligu: Study concept and drafting the work.
Acknowledgement- We acknowledge the contribution of Dr Aridita Datta for helping us in writing the manuscript of this paper.

\section{Funding: Nil, Conflict of interest: None} Permission of IRB: Yes

\section{References}

1. Ferlay J, Soerjomataram I, Dikshit R, Eser S, Mathers $\mathrm{C}$, Rebelo $\mathrm{M}$ et al. Cancer incidence and mortality worldwide: Sources, methods and major patterns in GLOBOCAN 2012. Int. J. Cancer.2015 Mar;136(5): E359-86.doi: 10.1002/ijc.29210.

2. Arnold M, Sierra MS, Laversanne M, Soerjomataram I, Jemal A, Bray F. Global patterns and trends in colorectal cancer incidence and mortality. Gut.2017;66 (4): 683-91. doi: 10.1136/gutjnl-2015-310912

3. Martın-Lopez JE, Beltran-Calvo C, Rodrıguez-Lopez R, Molina-Lopezl T. Comparison of the accuracy of CT colonography and colonoscopy in the diagnosis of colorectal cancer. Colorectal Disease. 2014 Mar; 16(3): O82-9.doi: 10.1111/codi.12506.

4. Day JJ, Freeman AH, Coni NK, Dixon AK. Barium enema or computed tomography for the frail elderly patient? Clin Radiol .1993 Jul; 48: 48-51.doi: https:// doi.org/10.1016/S0009-9260(05)80108-4

5. Bor R,Fabian A,Szepes Z. Role of ultrasound in colorectal diseases. World J Gastroenterol. 2016 Nov; 22 (43): 9477-87.doi: 10.3748/wjg. v22.i43.9477.

6. Shirahama M, Koga T, Ishibashi H, Uchida S, Ohta Y. Sonographic features of colon carcinoma seen with high-frequency transabdominal ultrasound. J Clin Ultrasound.1994 Jul-Aug; 22(6):359-65.https://doi.org/ $10.1002 /$ jcu. 1870220602 .

7. Balthazar E J, Megibow A J, Hulnick D, Naidich DP. Carcinoma of the colon: Detection and preoperative staging by CT. AJR. 1988 Feb ; 150(2):301-6.DOI: 10. 2214/ajr.150.2.301

8. Owens AP, Banerjee B, Morewood DJW: Sonography as an aid to diagnosis of caecal carcinoma in the elderly. Clin Radiol. 1983 Nov; 34:669-72.

9. Sianesi M, Rossi A, Miselli A, Farinon AM. Ultrasonic detection of colonic carcinoma in emergency. Dis Colon Rectum.1984 Mar; 27(3): 168-71. 


\section{Original Research Article}

10. Khoo HT. The ultrasonic demonstration of colonic carcinoma. Aust Rdiol .1981 Mar; 25(1):23-30.

11. Price J, Metreweli C. Ultrasonographic diagnosis of clinically non-palpable primary colonic neoplasms. BJR. 1988;61(723):190-5.DOI: 10.1259/0007-1285-61723-190.

12. Hirooka N, Ohno T, Misonoo M, Kobayashi C, MushaH,. Mori H, et al. Sono-enterocolonography by oral water administration. J Clin Ultrasound.1989 Oct; 17 (8): 585-9.

13. LimbergB.Diagnosis of large bowel tumors by colonic sonography. Lancet.1990 Jan; 335(8682):144-6.

14. Limberg B. Diagnosis and staging of colonic tumors by conventional abdominal sonography as compared with hydrocolonic sonography. N Engl J Med. 1992; 327:65-9.

15. Chui DW, Gooding GA, McQuaid KR,Griswold V,Grendell JH. Hydrocolonic ultrasonography in the detection of colonic polyps and tumors. N Engl J Med.1994 Dec; 331 (25):1685-8.DOI: 10.1056/NEJM 199412223312504

16. W K Loftus, C Metreweli, Sung JJ, Yang WT, Leung VK, Set PA.Ultrasound, CT and colonoscopy of colonic cancer. Br JRadiol.1999 Feb;72(854):144-8.doi: 10.1259/bjr.72.854.10365063.

17. Fink, M., Freeman AH, Dixon AK,Coni NK. Computed tomography of the colon in elderly people. Br Med J. 1994; 308(6935):1018.

18. Loftus WK, Metreweli C, Sung JJ, Yang WT, Leung VK, Set PA. Ultrasound, CT and colonoscopy of colonic cancer. Bri J Radiol. 1999 Feb; 72(854):144-8.
19. Okizuka H, Sugimura K, Shinozaki N, Watanabe K. Colorectal carcinoma: evaluation with ultrafast CT. Clin Imaging.1995 Oct-Dec; 19(4):247-51.

20. Amin Z, Boulos PB, Lees WR. Technical report: spiral CT pneumocolon for suspected colonic neoplasms. Clin Radiol. 1996 Jan; 51(1):56-61.

21. Hara AK, Johnson CD, Reed JE, Ahlquist DA, Nelson H, Ehman RL, McCollough CH, Ilstrup DM. Detection of colorectal polyps by computed tomographic colography: feasibility of a novel technique. Gastroenterology.1996 Jan; 110 (1): 284-90.

22. Macari M, Berman P, Dicker M, Milano M, Megibow AJ. Usefulness of CT colonography in patients with incomplete colonoscopy. AJR Am J Roentgenol.1999 Sep; 173(3):561-4. DOI: 10.2214/ ajr. 173.3.10470879.

23. Morrin MM, Kruskal JB, Farrell RJ, Goldberg SN, McGee JB, Raptopoulus V. Endoluminal CT colonography after incomplete endoscopic colonoscopy. AJR Am J Roentgenol .1999 Apr; 172:913-8.doi: 10.2214/ ajr. 172.4.10587120

24. Fenlon HM, McAneny DB, Nunes DP, Clarke PD, Ferrucci JT. Occlusive colon carcinoma: virtual colonoscopy in the preoperative evaluation of the proximal colon. Radiology. 1999 Feb; 210(2):423-8. DOI: 10.1148/radiology.210.2. r99fe21423.

25. Rex DK, Cutler CS, Lemmel GT, Rahamani EY, Clark DW, Helper DJ, et al.Colonoscopic miss rates of adenomas determined by back-to-back colonoscopies. Gastroenterology.1997 Jan; 112(1):24-8.

\section{How to cite this article?}

Sumnyan W, Komut O, Ligu L. Comparison of high-frequency transabdominal ultrasonography and spiral computed tomography with colonoscopy in diagnosis of colon cancer. Int J Med Res Rev 2018; 6(07):341-347. doi:10.17511/ijmrr.2018.i07.01. 